\title{
Effects of sucrose on anthocyanin production in hypocotyl of two radish (Raphanus sativus) varieties
}

\author{
Masakazu Hara*, Karin Oki, Kyoko Hoshino, Toru Kuboi \\ Faculty of Agriculture, Shizuoka University, 836 Ohya, Shizuoka 422-8529, Japan \\ *E-mail: masahara@agr.shizuoka.ac.jpＴel: +81-54-238-5134, Fax: +81-54-238-4881
}

Received June 10, 2004; accepted August 31, 2004 (Edited by K.Yazaki)

\begin{abstract}
Variations in swollen hypocotyl of red and white radish (Raphanus sativus) varieties are attributed to the level of anthocyanin accumulation. However, it is not known which genes in the biosynthetic pathway are involved in this variation. Upon treatment with exogenous sucrose, hypocotyl of red radish produced a large amount of anthocyanins, while that of white radish produced little. Six genes involved in anthocyanin production and one gene in lignin biosynthesis in red and white varieties were tested to demonstrate which genes were expressed on addition of sucrose. In red radish, transcripts of all six anthocyanin-related genes were remarkably induced by sucrose, while none of them were induced in white radish. Transcripts for cinnamoyl-CoA reductase involved in lignin biosynthesis were not influenced by sucrose in either variety. These results suggest that the low level of anthocyanin accumulation in white radish is due to the weak response of anthocyanin-related genes to sucrose.
\end{abstract}

Key words: Anthocyanin, Raphanus sativus.

Anthocyanins are natural pigments found in many plant species and are postulated to have various functions in the growth of plants. Anthocyanins protect tissue from UV irradiation (Holton and Cornish 1995). Autumn leaves produce anthocyanins to prevent photo-oxidative damage as leaves senesce (Feild et al. 2001). Anthocyanins in petals attract pollination and those in fruits help with seed dispersal (Holton and Cornish 1995). Light, which is the most important environmental cue for anthocyanin biosynthesis, stimulates signal transduction and gene expression involved in the biosynthesis (Mol et al. 1996). In addition, the accumulation of anthocyanins is enhanced by addition of sugar (Tsukaya et al. 1991), phosphate limitation (Dixon and Paiva 1995), and cold stress (Leyva et al. 1995).

Sugar is a major enhancer of both anthocyanin production and organ development in reproductive organs like flowers. An elevation in the sugar concentration and the phosphorylation of sugars by hexokinase were necessary for pigmentation and petal development (Weiss 2000). The genes involved in the biosynthesis of anthocyanins were activated by the addition of sucrose in reproductive organs. Expression of the chalcone synthase $(\mathrm{CHS})$ gene derived from petunia petals in transgenic Arabidopsis leaves was also induced by administration of sugars (Tsukaya et al. 1991). Petunia and Arabidopsis chs genes possess "sucrose boxes", which were found in sucrose-responsive genes such as the sporamin and $\beta$-amylase genes, in the $5^{\prime}$ flanking regions (Tsukaya et al. 1991). Anthocyanin production in Vitis vinifera berries and cell cultures was enhanced by sugars. The expression of seven genes involved in anthocyanin biosynthesis was enhanced during the development of berry skins in $V$. vinifera (Boss et al. 1996). The magnitude of expression of the anthocyanin-related genes was correlated with the accumulation of sugars during the development of berries in $V$. vinifera. The production of anthocyanin was markedly increased by sucrose in $V$. vinifera cells. Calcium ion, protein kinases, and protein phosphatases were involved in this process (Vitrac et al. 2000). The application of sucrose to $V$. vinifera cells up-regulated the expression of genes for dihydroflavonol reductase $(D F R)$ and anthocyanidin synthase (ANS) (Gollop et al. 2002; Gollop et al. 2001).

Sugar-inducible anthocyanin production has been characterized extensively in flowers and berries, but little in vegetative tissues. Anthocyanin production in Arabidopsis was enhanced when plants were grown on a sucrose-containing medium (Ohto et al. 2001). Swollen hypocotyls of radish (Raphanus sativus) produce anthocyanins. Sugar content and anthocyanin accumulation showed a positive correlation during the swelling of hypocotyls (Hara et al. 2003). Detached

Abbreviations: ANS, anthocyanidin synthase; CCR, cinnamoyl-CoA reductase; CHI, chalcone flavanone isomerase; CHS, chalcone synthase; DFR, dihydroflavonol reductase; F3H, flavanone 3-hydroxylase; PAL, phenylalanine ammonia lyase; RT-PCR, reverse transcription-polymerase chain reaction. 
hypocotyls accumulated anthocyanin on the exogenous application of sugars. CHS and ANS gene expression was promoted by sucrose in the detached-hypocotyl system (Hara et al. 2003). Deficient mutants could be a convenient tool for elucidating the regulatory mechanism of anthocyanin production in response to sucrose. In this paper, we investigated the production of anthocyanins and regulation of anthocyanin-related genes by sucrose in red and white varieties of radish.

A detached hypocotyl system (Hara et al. 2003) was used to indicate whether red and white varieties of radish produce anthocyanins. Two cultivars, Comet and Icicle, were used as red and white varieties, respectively. Radish seeds were germinated under sterile conditions in a growth chamber with a cycle of $10 \mathrm{~h}$ of light ( $c a$. $\left.50 \mu \mathrm{E} \mathrm{m}^{-2} \mathrm{~s}^{-1}\right)$ and $14 \mathrm{~h}$ of darkness at $25^{\circ} \mathrm{C}$. Four-dayold etiolated seedlings (hypocotyl length, 5 to $6 \mathrm{~cm}$ ) were cut and hypocotyls were excised. The hypocotyls were laid on Murashige and Skoog plates containing $175 \mathrm{mM}$ sucrose in 9-cm diameter glass dishes. The sucrose concentration was optimized for maximum anthocyanin accumulation in red radish (Hara et al. 2003). Samples were incubated at $25^{\circ} \mathrm{C}$ under constant illumination ( $c a$. $\left.50 \mu \mathrm{E} \mathrm{m}^{-2} \mathrm{~s}^{-1}\right)$. The cultivation period was six days. Sampling was done every day. Radish anthocyanins are mixtures of twelve acylated pelargonidin glycosides (Otsuki et al. 2002; Giusti et al. 1999). Anthocyanins in hypocotyl were extracted with $0.1 \mathrm{~N} \mathrm{HCl}$. Anthocyanin content was calculated using the molar extinction coefficient of raphanusins $(\varepsilon=31760$ at $511 \mathrm{~nm})$ (Ishikura and Hayashi 1963). Fresh weights and anthocyanin levels in hypocotyls of red and white radish were compared (Figure 1). During the cultivation, fresh weight was constant and unaffected by sucrose in either variety (Figure 1A, C). Anthocyanin production was enhanced by sucrose in the hypocotyl of red radish. The content reached $c a .360 \mathrm{nmol} / \mathrm{g}$ fresh weight on sucrose treatment for six days (Figure 1B). The anthocyanin content was low ( $c a .50 \mathrm{nmol} / \mathrm{g}$ fresh weight) in the control hypocotyl. It is likely that this weak induction is promoted by light. Hypocotyls of white radish, however, produced little anthocyanin even when sucrose was added (Figure 1D). Two hypotheses were postulated to explain this phenomenon in white radish. First, the genes for the biosynthesis of anthocyanin are mutated and their functions lost. Second, the gene expression was strongly inhibited though the genes themselves were fully functional. The second hypothesis seems plausible, since the hook of white radish seedlings produced anthocyanins at the early stage of germination under light (data not shown).

Radish produces acylated pelargonidin glycosides through the biosynthetic pathway outlined in Figure 2. In this study, phenylalanine ammonia lyase (PAL), CHS, chalcone flavanone isomerase $(\mathrm{CH} I)$, flavanone 3-
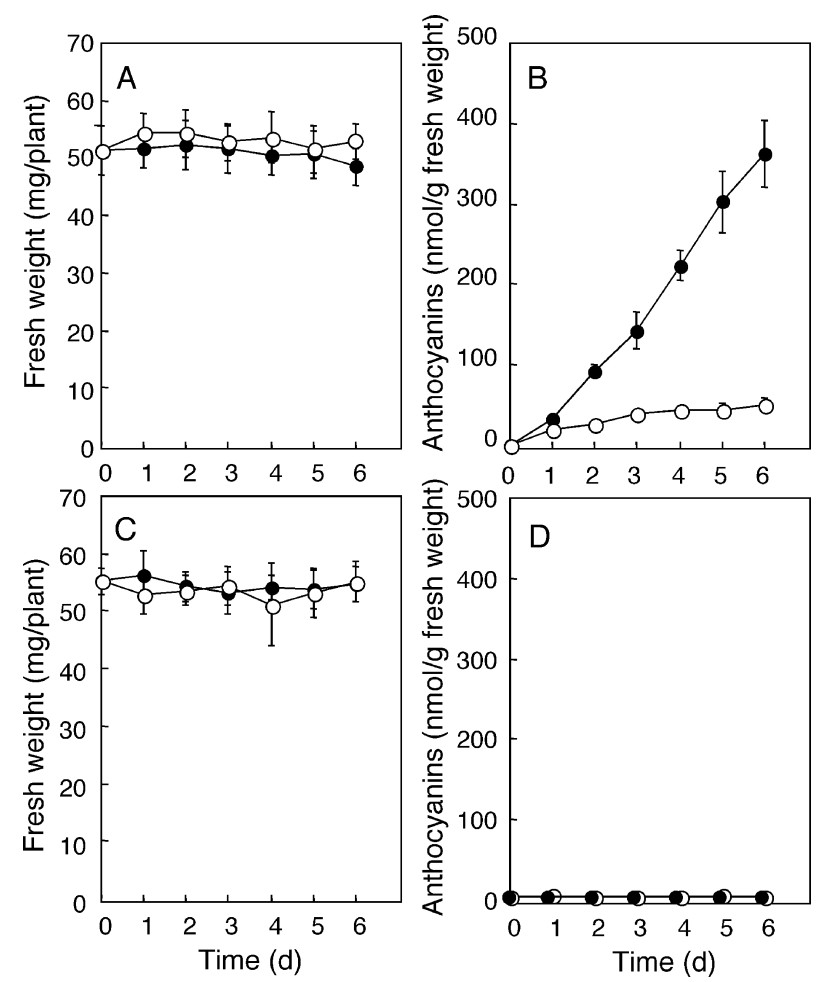

Figure 1. Effects of sucrose on fresh weight $(\mathrm{A}, \mathrm{C})$ and anthocyanin production $(\mathrm{B}, \mathrm{D})$ in hypocotyl of red $(\mathrm{A}, \mathrm{B})$ and white $(\mathrm{C}, \mathrm{D})$ radish. Period of incubation was 6 days. Open and closed circles indicate treatment with no sucrose and $175 \mathrm{mM}$ sucrose, respectively. The plotted data and error bars indicate the means and variations of duplicate individual experiments.

hydroxylase $(F 3 H), D F R, A N S$, and cinnamoyl-CoA

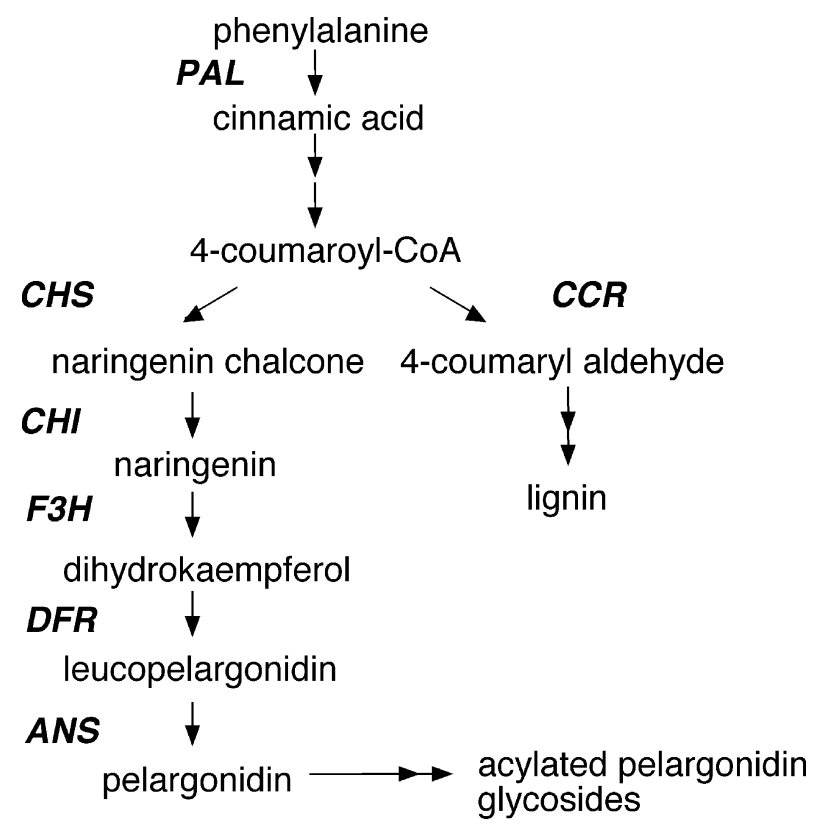

Figure 2. Postulated biosynthetic pathway of radish anthocyanins. $P A L, C H S, C H I, F 3 H, D F R, A N S$, and CCR represent phenylalanine ammonia lyase, chalcone synthase, chalcone flavanone isomerase, flavanone 3-hydroxylase, dihydroflavonol reductase, anthocyanidin synthase, and cinnamoyl-CoA reductase, respectively. 
reductase $(C C R)$ were tested to determine which genes were activated by sucrose. Partial cDNAs for the corresponding genes were cloned by reverse transcription-polymerase chain reaction (RT-PCR) using RNA PCR Kit Ver. 2.1 (Takara, Tokyo, Japan). Details of $C H S$ and $A N S$ cloning were described previously (Hara et al. 2003). To isolate PAL, CHI, F3H, DFR, and cDNAs, primers were designed on the basis of conserved regions known in other species. The sequences of primers were as follows; sense primer (5'-CAYCAYCCNGGNCARATHGARGC-3') and antisense primer (5'TGYTGYTCIGCISWYTGIACRTG-3') for $P A L$, sense primer (5'-GCTTCCTCCAACACTCTCTTCC-3') and antisense primer (5'-AATCTCTCGGCAACCCTCAACC- $3^{\prime}$ ) for $\mathrm{CHI}$, sense primer ( $5^{\prime}$-ATHTTYCARGTNGTNGAYCAYGG-3') and antisense primer (5'-TGRTCIGCRTTYTTRAAICKICC-3') for $F 3 H$, sense primer ( $5^{\prime}$ ATGGAYTTYGARWSNAARGAYCC- $3^{\prime}$ ) and antisense primer ( $5^{\prime}$-TCNARRTGNACRWAYTGNCCYTG- $\left.3^{\prime}\right)$ for $D F R$, and sense primer ( $5^{\prime}$-ACIGGIGCIGSIGGITWYATHGC-3') and antisense primer ( $5^{\prime}$-GGRTAYTCNGGRAARAAYTTNGC-3') for $C C R$. PCR products were sequenced and identified as radish homologues for the corresponding genes. Accession numbers were $\mathrm{AB} 087212, \mathrm{AB} 087208, \mathrm{AB} 087211, \mathrm{AB} 087210$, and $\mathrm{AB} 087207$ for $P A L, C H I, F 3 H, D F R$, and $C C R$,
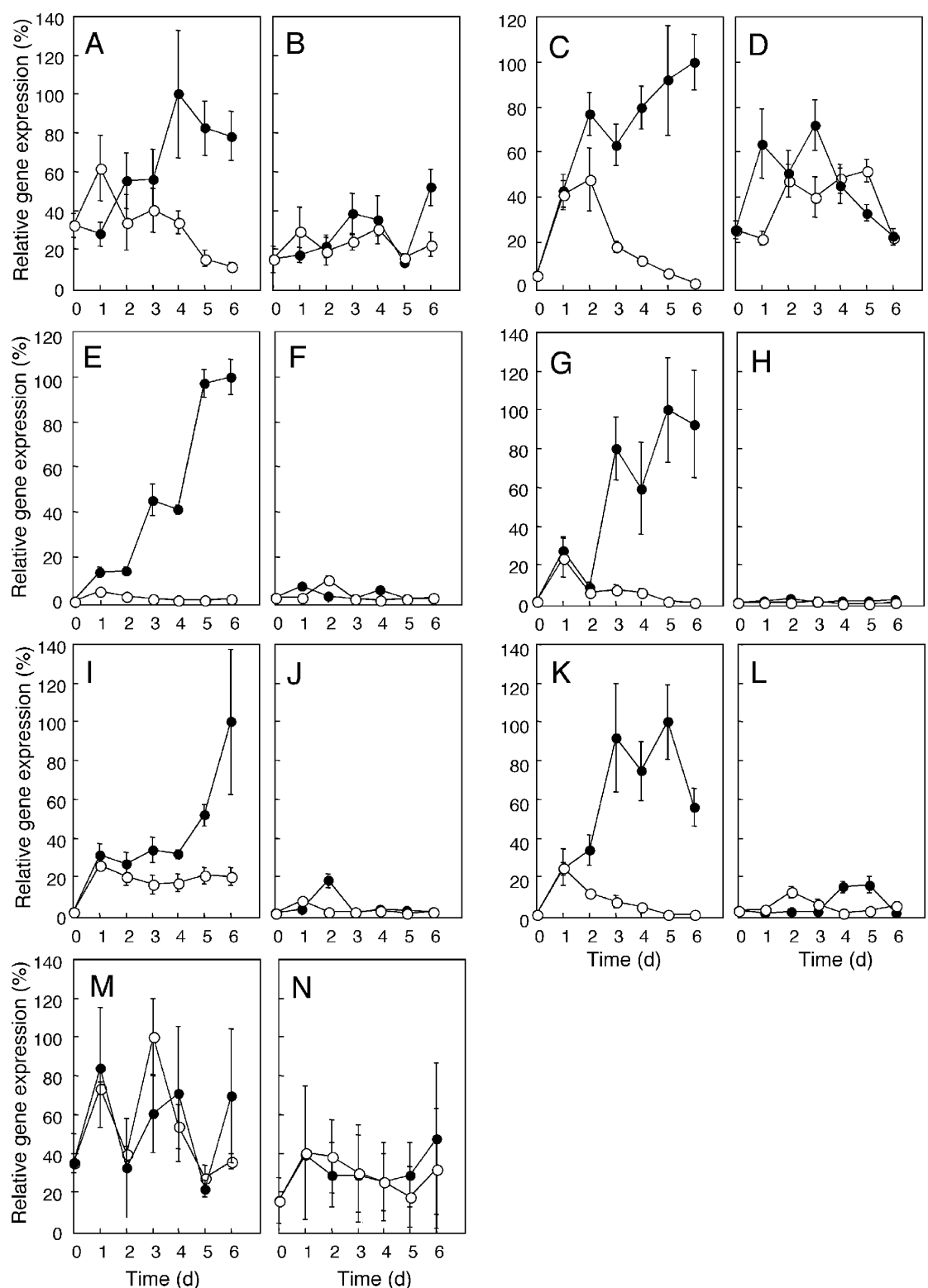

Figure 3. Effects of sucrose on PAL (A, B), CHS (C, D), CHI (E, F), F3H (G, H), DFR (I, J), ANS (K, L), and CCR (M, N) expression in hypocotyl of red (A, C, E, G, I, K, and M) and white (B, D, F, H, J, L, and N) radish. Gene expression was measured by the quantitative RT-PCR method described in the text. The highest mean value in each graph was set to $100 \%$. Open and closed circles indicate treatment with no sucrose and $175 \mathrm{mM}$ sucrose, respectively. Period of incubation was 6 days. The plotted data and error bars indicate the means and variations of duplicate individual experiments. 
respectively. The expression of each gene was measured by semiquantitative RT-PCR (Goidin et al. 2001) using a QuantumRNA 18S Internal Standards Kit (Ambion, TX, USA). In this assay system, the relative expression of the target gene was estimated as the ratio of the target gene expression against rRNA expression. The sequences of primers were as follows; sense primer $\left(5^{\prime}\right.$-CTCGTAACCGGATTGGCTAGG- $\left.3^{\prime}\right)$ and antisense primer $\left(5^{\prime}\right.$-AGCTCGTACATGAAGTTAGCG-3') for $P A L$, sense primer (5'-GCTTCCTCCAACACTCTCTTCC-3') and antisense primer $\left(5^{\prime}\right.$-AATCTCTCGGCAACCCTCAACC-3') for $C H I$, sense primer (5'-TTGCTCAGATAGTGGCCATGG- $3^{\prime}$ ) and antisense primer ( $5^{\prime}$-TTTGGTGGCGGATATGACTCG-3') for $F 3 H$, sense primer $\left(5^{\prime}\right.$-TGCCTCGTTACGAGTGATAGG-3') and antisense primer (5'-CGACAGTGAATGGAGTGTTGG-3') for $D F R$, and sense primer ( $5^{\prime}$-ATCTCAACAACTTCGCCGCGG-3') and antisense primer (5'-AGAGAACTAGAAGGGGCCAAGG-3') for $C C R$. Primers for $C H S$ and $A N S$ were noted previously (Hara et al. 2003). After the band intensity of PCR products was determined using NIHImage software, the relative mRNA content was given as the intensity of target PCR products/that of rRNA PCR products according to the instruction manual of the QuantumRNA Kit. The highest mean value in each timecourse was set to $100 \%$. In the red radish, PAL and $C H S$ expression was enhanced on the addition of sucrose (Figure 3A, C). However, their expression levels in the white radish were not influenced by sucrose (Figures 3B, D). $C H I, F 3 H, D F R$, and $A N S$ were remarkably expressed on addition of sucrose in the red radish (Figures 3E, G, I, K). On the other hand, the expression of these genes was strongly suppressed in the white variety despite the addition of sucrose (Figures $3 \mathrm{~F}, \mathrm{H}, \mathrm{J}$, $\mathrm{L})$. The expression of $C C R$ located at the branching point leading to lignin biosynthesis was not altered by sucrose in either variety (Figures $3 \mathrm{M}, \mathrm{N}$ ).

A summary of the data in Figure 3 is shown in Figure 4. The gene expression of PAL, CHS, CHI, F3H, DFR, and $A N S$ was enhanced by sucrose in red radish. Notably, the latter four genes specific to the biosynthesis of anthocyanins were remarkably activated. In the white radish, the four anthocyanin-specific genes were expressed very weakly and did not respond to sucrose. It is known that transcription factors cooperatively regulate the genes involved in anthocyanin production (Mol et al. 1996). Such transcription factors may be strongly expressed when hypocotyl of red radish is treated with sucrose. Since $C H I, F 3 H, D F R$, and $A N S$ expression was simultaneously suppressed, it is postulated that sucroseresponsive transcription factors were not expressed even when sucrose was added, or mutations occur in one of these factors. A study of the transcription factors will provide crucial information about the regulatory mechanism of sucrose-inducible anthocyanin biosyn-
Red radish

White radish

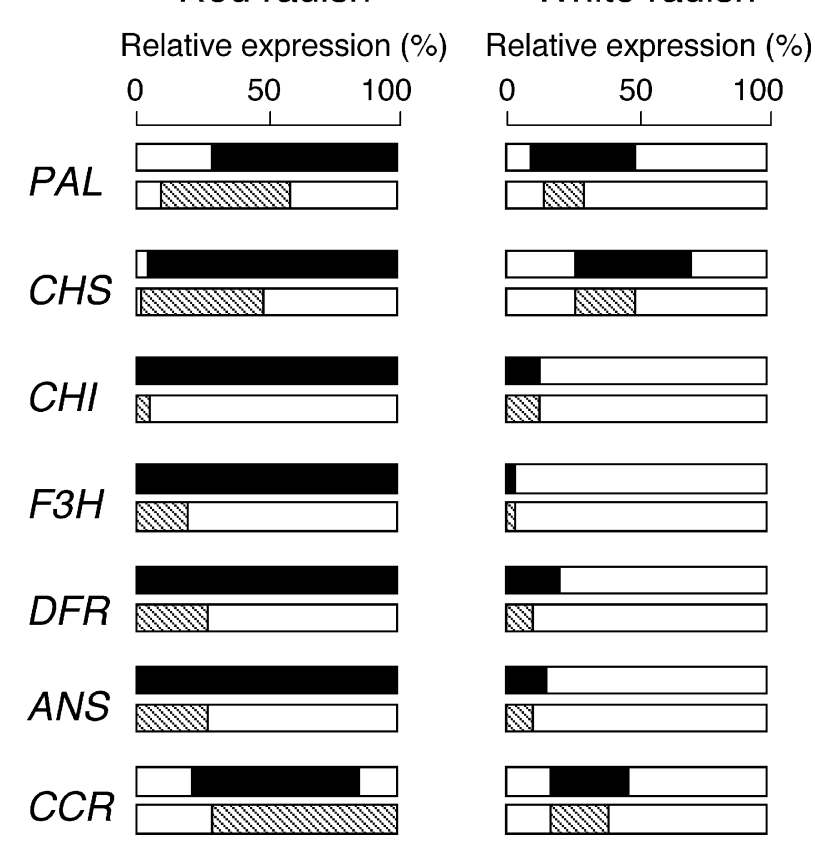

Figure 4. Summary of sucrose-regulated expression of anthocyanin biosynthetic genes in red and white radish. The figure was prepared from the results of Figure 3. Bars indicate the range of the expression level for each gene. Closed and shaded bars represent relative gene expression in hypocotyl treated with $175 \mathrm{mM}$ sucrose and no sucrose, respectively.

thesis.

\section{Acknowledgements}

This article is dedicated to the late Emeritus Professor Mamoru Tabata.

\section{References}

Boss PK, Davies C, Robinson SP (1996) Analysis of the expression of anthocyanin pathway genes in developing Vitis vinifera $\mathrm{L}$. cv Shiraz grape berries and the implications for pathway regulation. Plant Physiol 111: 1059-1066

Dixon RA, Paiva NL (1995) Stress-induced phenylpropanoid metabolism. Plant Cell 7: 1085-1097

Field TS, Lee DW, Holbrook NM (2001) Why leaves turn red in autumn. The role of anthocyanins in senescing leaves of redosier dogwood. Plant Physiol 127: 566-574

Giusti MM, Rodriguez-Saona LE, Wrolstad RE (1999) Molar absorptivity and color characteristics of acylated and nonacylated pelargonidin-based anthocyanins. J Agr Food Chem 47: 4631-4637

Goidin D, Mamessier A, Staquet M-J, Schmitt D, Berthier-Vergnes O (2001) Ribosomal 18S RNA prevails over glyceraldehyde3 -phosphate dehydrogenase and $\beta$-actin genes as internal standard for quantitative comparison of mRNA levels in invasive and noninvasive human melanoma cell subpopulations. Anal Biochem 295: 17-21

Gollop R, Even S, Colova-Tsolova V, Peri A (2002) Expression of the grape dihydroflavonol reductase gene and analysis of its promoter region. JexpBot 53: 1397-1409 
Gollop R, Farhi S, Peri A (2001) Regulation of the leucoanthocyanidin dioxygenase gene expression in Vitis vinifera. Plant Sci 161: 579-588

Hara M, Oki K, Hoshino K, Kuboi T (2003) Enhancement of anthocyanin biosynthesis by sugar in radish (Raphanus sativus) hypocotyl. Plant Sci 164: 259-265

Holton TA, Cornish EC (1995) Genetics and biochemistry of anthocyanin biosynthesis. Plant Cell 7: 1071-1083

Ishikura N, Hayashi K (1963) Chromatographic separation and characterization of the component anthocyanins in radish root. Bot Mag Tokyo 76: 6-13

Leyva A, Jarillo JA, Salinas J, Martinez-Zapater JM (1995) Low temperature induces the accumulation of phenylalanine ammonia-lyase and chalcone synthase mRNA of Arabidopsis thaliana in a light-dependent manner. Plant Physiol 108: $39-46$

Mol J, Jenkins GI, Schaefer E, Weiss D (1996) Signal perception, transduction, and gene expression involved in anthocyanin biosynthesis. Crit Rev Plant Sci 15: 525-557

Ohto M, Onai K, Furukawa Y, Aoki E, Araki T, Nakamura K (2001) Effects of sugar on vegetative development and floral transition in Arabidopsis. Plant Physiol 127: 252-261

Otsuki T, Matsufuji H, Takeda M, Toyoda M, Goda Y (2002) Acylated anthocyanins from red radish (Raphanus sativus L.). Phytochem 60: 79-87

Tsukaya H, Ohshima T, Naito S, Chino M, Komeda Y (1991) Sugar-dependent expression of the CHS-A gene for chalcone synthase from petunia in transgenic Arabidopsis. Plant Physiol 97: 1414-1421

Vitrac X, Larronde F, Krisa S, Decendit A, Deffieux G, Merillon JM (2000) Sugar sensing and $\mathrm{Ca}^{2+}$-calmodulin requirement in Vitis vinifera cells producing anthocyanins. Phytochem 53: 659-665

Weiss D (2000) Regulation of flower pigmentation and growth: multiple signaling pathways control anthocyanin synthesis in expanding petals. Physiol Plant 110: 152-157 